\title{
XXXV. On the specific heat of the gases
}

\section{W.T. Haycraft Esq.}

To cite this article: W.T. Haycraft Esq. $(1824)$ XXXV. On the specific heat of the gases, Philosophical Magazine Series 1, 64:317, 200-207, DOI: $10.1080 / 14786442408644586$

To link to this article: http://dx.doi.org/10.1080/14786442408644586

$$
\text { 曲 Published online: } 27 \text { Jul } 2009 .
$$

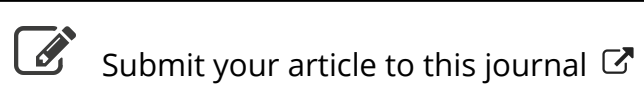

\footnotetext{
Џ Article views: 2
}

Q View related articles $\sqsubset$ 
In general it appears, too, from ideas that have occurred to me, of which I may say more hereafter, that any equation, as

$$
\mathrm{F}\left\{x, \psi x, \psi \alpha x, \psi \alpha^{2} x, \ldots \psi \alpha^{n} x\right\}=0
$$

has $n$ arbitrary functions, provided however that $a^{r} x=x$ and $r>n$; and that an equation of the form

$$
\mathrm{F}\left\{x, \psi x, \psi^{2} x, \ldots \psi^{n} x\right\}=0
$$

has the same number. My ideas, however, on this subject are not sufficiently matured to enable me to speak confidently.

The method of substitution pursued in our solution of (i) which is taken from Mr. Babbage's solution of $\psi x=\psi \alpha x$, applies very successfully even in cases.where it is introduced but partially. Thus in the equation

$$
1+f x\{\psi x+\psi \alpha x\}=\psi x \cdot \psi \alpha x
$$

which Mr. Babbage has solved by a very neat process of differentiation and integration, but ultimately bringing out a transcendental solution, it may be applied partially to the evolution of an algebraic solution. By changing $x$ into $\alpha x$, we find that $f x=f a x$ when $a^{2} x=x$, which is the condition of the problem. For $\psi x$ on the left side only of the equation substitute $v \psi x+b \phi x$, and equate the result with the same result, having $x$ changed into $\alpha x$; and we shall find

$$
\psi \alpha x=\psi x+\phi x-\phi \alpha x,
$$

which substituted for $\psi \alpha x$ in (8) gives ultimately

$$
\psi x=\frac{1}{2} \sqrt{(\phi x-\phi \alpha x)^{2}+4\left(f x^{2}+1\right)}+f x-\frac{1}{2}(\phi x-\phi \alpha x)
$$

for the complete solution of (8).

Had we substituted for $\psi x$ on the right side only of (8), we should have found

and therefore

$$
\psi \alpha x=\frac{\varphi \alpha x}{\varphi x} \cdot \psi x
$$

$$
\psi x=\frac{1}{2} \sqrt{\frac{4 \phi x}{\varphi \alpha x}+\left(\frac{\phi x}{\varphi \alpha x}+1\right)^{2} f x^{2}}+\frac{f_{x}}{2}\left(\frac{\phi x}{\varphi \alpha x}+1\right)
$$

which is a solution equally general, but not so simple, as the preceding.

XXXV. On the Specific Heat of the Gases. By W. T. HAXCraFT, Esq.*

THE experiments which $\mathrm{I}$ now submit to the Royal Society are repetitions of those I made many months ago, for the purpose of ascertaining the specific heats of the gases.

* From the Transactions of the Royal Society of Edinburgh, vol. $x$. 
The importance of the subject so impressed my mind, that I determined to spare no pains in the prosecution of the inquiry, and therefore I willingly withheld my first experiments from the public eye, until, by a fresh series, I might present them with the greater confidence. The apparatus employed in these experiments was calculated to operate upon greater quantities of the gases than the former one, and as every precaution which had been suggested was adopted, they have, perhaps, given even more decisive results than the last. The results themselves, however, are in every important particular exactly the same. It is also but justice to myself to state, that the conclusions which the former experiments led to, were exactly the reverse of what $I$ had anticipated, and that they seemed at the time totally opposed to the doctrines of Black and Crawford, which I am still disposed to credit to a limited degree.

Before I enter into the detail, it will be necessary to take notice of the modes in which former experimenters have proceeded in these inquiries, and to point out what I conceive to have been the sources of fallacy in some of their conclusions. Of all these modes, none were more elegant than that adopted by Professor Leslie; but as he himself states that their results were discordant with each other, it seems unnecessary to enter into a description of it. Dr. Crawford's method cunsisted of inclosing two different gases (previously exposed to muriate of lime, for the purpose of depriving them of their watery vapour,) in two close vessels of equal size and weight; these being heated to exactly the same temperature, by a very ingenious contrivance, were at the same time plunged into two vessels, containing water of a lower temperature: these vessels were also of the same size, form, and weight: then, by means of accurately adjusted thermometers, he ascertained the comparative rise of temperature occasioned by the two gases, and hence he determined their specific heats.

- I know of no imperfection in this mode, excepting that the quantities of the gases were so small, that the results could not be obtained with sufficient accuracy.

This defect is entirely obviated by the method adopted by Messrs. De la Roche and Berard: their apparatus consisted of a column of water, so adjusted as to act with a constant and equal pressure in a close vessel containing air; which being gradually expelled by the superincumbent water, pressed on the outward surface of a bladder containing the gas whose capacity was to be examined. From this bladder the gas was propelled through the calorimeter: this consisted of a vesse] containing water of a low temperature, through which a spiral Vol. 64. No. 317. Sept. 1824. 
tube passed to conduct the gas. Previous, however, to its entering the calorimeter, the gas was heated, by a particular contrivance, to the boiling temperature. After leaving the calorimeter, it was conducted, by means of turn-cocks, into another bladder; the latter was acted upon in the same way as the former. By means of this reciprocating action, Messrs. De la Roche and Berard could cause 225.2 cubic inches of gas, heated to the boiling temperature, to pass through the calorimeter every minute. The temperature communicated was ascertained by a thermometer, and from comparative trials the capacity of the different gases was inferred.

This last method was superior to that of Dr. Crawford, inasmuch as greater quantities of gas could be employed. In other respects it was far inferior, because the experiments were not, strictly speaking, comparative. Atmospheric air, whose capacity was their standard of comparison, was subjected to trial, and the results were remarked. The other gases, at different periods, with the surrounding media of different temperatures, and under different barometrical pressures, were examined; this plan involved endless and very difficult calculations, in order to adjust those differences. But the greatest imperfection in those experiments, was the neglect of depriving the gases of their watery vapours previous to their examination. The apparatus itself would not admit of this, because the water employed in the process would necessarily keep the gas and the whole apparatus in a state of moisture. Besides, this very great source of error was materially increased by the high temperature to which the gases were exposed, being a condition in which they are disposed to unite with a greater quantity of watery vapour than at ordinary temperatures. Considering the subject in this point of view, therefore, the experiments of Messrs. De la Roche and Berard may be supposed to determine the capacities of the different gases united with watery vapour at the boiling point, but by no means of those gases in their dry state, and at ordinary temperatures.

The apparatus now to be described will perhaps be found to unite the advantages and avoid the defects of both methods.

It consisted of two hollow brass cylinders (see Plate II.), in each of which was a piston attached to a spindle by means of two levers of equal length ; to the spindle was attached another lever, terminating in a handle, to be moved by an assistant. Each cylinder was closed at each end, excepting where the tubes were attached, which served to conduct the gases. By means of four valves to each cylinder, fixed in such a way as, though difficult to describe in writing, may be easily understood by reference to Plate II., each action of the piston forced 
forced a quantity of air through the tubes; thus, by means of one additional valve, the apparatus would act upon exactly twice the quantity of air that could be acted upon in a pump of the ordinary construction.

The pipes immediately connected with the four valves terminated in two tubes; through one of which the air, during the action of the apparatus, was propelled with a constant and almost uniform current, while, through the other, the same air having passed through the heating apparatus and the calorimeter, returned to the cylinder, to be acted upon again in the same way. The heating apparatus just mentioned consisted of a metallic vessel, about 16 inches long, containing hot water, through which the tubes passed, containing the air propelled from the cylinders : those tubes traversed the heating vessel three times before their exit, more effectually to secure the gases arriving at the temperature of the water contained in the vessel. By means of a lamp placed under this vessel, I could raise the temperature of the water to any point required. This last arrangement, however, was rather a matter of convenience than necessity, as it will be easily perceived that, from the mode of conducting the experiments, a fixed point of temperature was not required.

There were also two calorimeters, similar in construction to those of Messrs. De la Roche and Berard before described. Each of these was connected with the tube containing the gas, propelled by its cylinders through the heating apparatus, and likewise with that through which the air flowed to the cylinder; these tubes were all of metal, and air-tight.

The apparatus, then, must be considered as consisting of two distinct parts, exactly the counterparts of each other, each conveying an equal quantity of gas through the same heating medium, but through separate calorimeters.

The tubes communicating between the heating vessel and the calorimeters were one inch in length. In these tubes there was an opening, through which could be introduced a delicate thermometer, for the purpose of ascertaining the temperature of the gases as they entered the calorimeters.

Each of the calorimeters was inclosed in a polished metallic case, for the purpose of preventing, as much as possible, the absorption or escape of caloric during the process. These latter were also placed in a box containing water, which was repeatedly agitated, that the calorimeters might not be affected by the unequal temperatures of the walls of the apartment.

For the purpose of facilitating the operation of filling the apparatus with the gas operated upon, there was a turn-cock fixed in the course of each returning tube, by which the cur-

$$
\text { C c } 2
$$


rent of gas through the tube was interrupted. Two smaller turn-cocks also were fixed in the same tube, one on each side of the larger turn-cocks: these, when open, communicated with the external atmosphere. When, therefore, the large turn-cock was closed, and the small ones open, the air would necessarily, during the action of the machine, rush in at one of the small turn-cocks, and be forced out of the other, so that the air contained in the apparatus would be constantly renewed. In order, then, to fill the machine with the gas, nothing more was necessary than to form a connection between the gasometer or receiver containing the gas and the apparatus, by means of a tube connected to the small turn-cock first mentioned, through which the air rushed in. In performing this operation, however, I usually made use of an airpump to exhaust the apparatus, and then opening the turncock communicating to the gasometer, filled it with the gas required : after this operation had been several times repeated, I found the gas contained in the machine to be nearly as pure as that contained in the gasometer.

By a slight consideration of this description of the apparatus, which may be deemed rather prolix, and by an inspection of the plate, it will be perceived that two gases, contained in the two parts of the machine, will be under circumstances precisely similar; the quantities of gas transmitted through the calorimeters in a given time will be the same; the temperature of the surrounding media and the barometrical pressure will be equal; the temperature also of the gases themselves must be the same, because they passed through the same heating medium. In fine, the size of the tubes, cylin. ders, calorimeters and valves, was the same in the two parts of the machine.

Therefore, the temperature communicated by the two gases submitted to a comparative trial, will be the direct ratio of their comparative capacities for caloric, provided there be no disproportionate escape by absorption in the calorimeters, arising from the different temperatures of surrounding bodies.

This source of fallacy was obviated by the arrangement of Count Rumford, who contrived that the temperature of the surrounding medium should be as much above that of the calorimeter at the beginning, as it was lower at the end of the experiments.

The quantity of gas propelled through the calorimeter was 12 cubic inches during the action of the piston. Those actions, as regulated by a second pendulum, which was suspended in the apartment, being 120 every minute, the whole quantity would be 1440 cubic inches of gas propelled through the ca- 
lorimeter every minute. There was no occasion, however, to take these quantities into the account, because they were precisely the same of each gas subjected to trial.

My thermometers were adjusted by Mr. Adie of Edinburgh. Each degree was divided into 5 parts, which were sufficiently large to be divided by the eye into 4 parts; so that the temperature could be ascertained to a 20th part of a degree, making allowance for the imperfection of all instruments. Each calorimeter was furnished with its thermometer, the bulb of which was placed equidistant from its four sides: two smaller ones were placed so as to ascertain the temperature of the gases before entering into or coming out of the calorimeter. One was attached to the heating vessel, and another to the vessel of water which served as the surrounding medium of the calorimeters.

Having filled both the calorimeters with water of the temperature of $42^{\circ}$, and the heating vessel with it at a temperature of about $180^{\circ}$, I admitted atmospheric air into each part of the apparatus. The pistons were put into motion, and continued till each of the calorimeters arrived at a temperature of $84^{\circ}$, with a variation of little more than one-twentieth part of a degree. Thus the temperature of the calorimeters was raised $42^{\circ}$ each, with a correction of $\frac{1}{8} \frac{1}{0}$ th part of the whole. Much greater allowances may very properly be made for the imperfections of the instruments. This experiment was designed to prove the accuracy of the apparatus, and was often repeated, at different periods, with the same event. I was assisted in the following experiments by my friend Dr. Clendinning, to whom I am much indebted for their success.

\section{Experiments on Carbonic Acid. \\ No. 1.}

The part of the apparains which I call $A$ was filled with carbonic acid, obtained from carbonate of lime; the part B with common air. In each of the cylinders was placed, in a proper receptacle, a quantity of very dry muriate of lime, for the purpose of perfectly freeing the gases from watery vapour. The calorimeters being filled with water at a temperature of $42^{\circ}$, and the heating vessels with water at $149^{\circ} \frac{1}{2} \frac{0}{2}$, the following results were obtained. 


$$
\begin{aligned}
& \text { Temperature of Calori. } \\
& \text { meter A, through } \\
& \text { which the Carbonic } \\
& \text { Acid passed. }
\end{aligned}
$$

At the beginning of experiment, $\} 42^{\circ} \mathrm{Fahr}$.

After 15 minutes, $68 \frac{2}{20}$

At the beginning of experiment, $42 \frac{1}{20}$

After 15 minutes, $66 \frac{1}{2} 0$

At the beginning of experiment, $\} 42$

After 40 minutes, $71 \frac{10}{2} 0$

At the beginning ? of experiment,

45

After 35 minutes, $68 \frac{5}{20}$

At the beginning of experiment, $\}$ $45 \frac{1}{2} \frac{4}{0}$

After 25 minutes, $63 \frac{5}{80}$
Temperature of Calorimeter $B$, through Air passed. Acid inferred from the comparative Rise of the Temperature of the Air being 10000 .

In these experiments it will be perceived, that the two first indicate that carbonic acid has a less capacity for caloric than common air. The three last, however, which do not differ materially from each other, will indicate an equal capacity, if we take the average of their results. ' The cause of the two first experiments indicating a lesser capacity, I suppose to arise from the gas not being perfectly freed from watery vapours. In the experiments I made last year, I observed that it was necessary to expose this gas to the drying influence of muriate of lime, for 35 minutes at least before it indicated the same specific heat as atmospheric air. This is not the case with all the other gases : from hence I would infer, that it has a greater affinity with watery vapour.

The gas contained in the gasometer, as indicated by limewater, contained 99 per cent. of carbonic acid; that taken from the apparatus after the experiments were concluded, by the same test, contained 90 per cent. The temperatures of the gases while entering the calorimeters were equal, as indicated by the thermometers. It is worthy of remark, however, that these temperatures appeared several degrees lower than that of the water contained in the heating apparatus through which they 
passed. This will be easily explained, when we consider that a thermometer can never indicate the true temperature of any gas or vapour, which is itself pervious to the radiation of heat or cold from surrounding bodies. On this account the thermometers indicated a temperature of the gases much lower than the true one, they being necessarily placed so near the calorimeters, which usually contained water of a temperature nearly $100^{\circ}$ lower than that of the gases. In the same manner, the gases issuing from the calorimeters appeared to have a temperature something lower than that of the calorimeters themselves, being surrounded with objects of a lower temperature than that of the calorimeter.

\section{Experiments on Oxygen Gas.}

No. 1.

Having filled the part $A$ with oxygen gas procured from the black oxide of manganese, and every arrangement being made as before, the following results were observed:

\begin{tabular}{|c|c|c|c|}
\hline & $\begin{array}{l}\text { emperature of Calori- } \\
\text { meter A, containing } \\
\text { Oxygen Gas. }\end{array}$ & $\begin{array}{c}\text { Of Calorimeter B, } \\
\text { containinge At. } \\
\text { mospheric Air. }\end{array}$ & Inferred Capaclties. \\
\hline $\begin{array}{l}\text { At the beginuing } \\
\text { of experiment, }\end{array}$ & $45^{\circ} \frac{6}{20}$ & $45^{\circ} \frac{5}{20}$ & \\
\hline After 5 minutes, & $61 \frac{1}{2} \frac{6}{0}$ & $61 \frac{1}{2} \frac{5}{0}$ & 10000 \\
\hline After 10 minutes, & $67 \frac{2}{20}$ & $67 \frac{1}{20}$ & 10000 \\
\hline After 15 minutes, & $71^{20}$ & $70 \frac{18}{2} \frac{8}{0}$ & 10019 \\
\hline After 20 minutes, & $74 \frac{9}{2} \pi$ & $74 \frac{9}{20}$ & 9982 \\
\hline
\end{tabular}

A No. 2. $\mathrm{B}$

$\begin{array}{llll}\begin{array}{c}\text { At the beginning } \\ \text { of experiment, }\end{array} & 56 \cdot 6^{\circ} & 56 \cdot 4^{\circ} & 10000 \\ \text { In 10 minutes, } & 66 \cdot 16 & 66 \cdot 14 & 10000 \\ \text { In 15 minutes, } & 71 & 70 \cdot 18 & 10000 \\ \text { In 20 minutes, } & 74 \cdot \frac{4}{20} & 74 \cdot \frac{2}{20} & 10000\end{array}$

The temperature of the gases entering the calorimeters was equal, being each $137^{\circ}$. The gas contained in the gasometer before the apparatus was filled, indicated $98^{\circ}$ per cent. of oxygen, by the test of sulphuret of lime. After the experiment was concluded, that contained in the apparatus indicated $91^{\circ}$ per cent.

[To be continued.] 